\title{
Liquid-gas phase transition in nuclear matter from realistic many-body approaches
}

\author{
A. Rios* \\ National Superconducting Cyclotron Laboratory and Department of Physics and Astronomy, Michigan State University, \\ East Lansing, 48824-1321 Michigan, USA \\ A. Polls and A. Ramos \\ Departament d'Estructura i Constituents de la Matèria and Institut de Ciències del Cosmos, Universitat de Barcelona, \\ Avda. Diagonal 647, E-08028 Barcelona, Spain \\ H. Müther \\ Institut für Theoretische Physik, Universität Tübingen, D-72076 Tübingen, Germany
}

(Received 15 May 2008; published 20 October 2008)

\begin{abstract}
The existence of a liquid-gas phase transition for hot nuclear systems at subsaturation densities is a wellestablished prediction of finite-temperature nuclear many-body theory. In this paper, we discuss for the first time the properties of such a phase transition for homogeneous nuclear matter within the self-consistent Green's function approach. We find a substantial decrease of the critical temperature with respect to the Brueckner-HartreeFock approximation. Even within the same approximation, the use of two different realistic nucleon-nucleon interactions gives rise to large differences in the properties of the critical point.
\end{abstract}

DOI: 10.1103/PhysRevC.78.044314

PACS number(s): 21.30.Fe, 21.65.Mn

\section{INTRODUCTION}

The study of the thermal properties of nuclear matter in terms of realistic many-body approaches has received little attention in the literature, in spite of its potential applications in astrophysics and in the physics of heavy ion collisions. Dense hadronic matter appears in some astrophysical scenarios, and it can be particularly hot in the very early stages after the creation of a protoneutron star in a type-II supernova explosion. Typical temperatures for such systems are in the range of 20-60 MeV [1]. It is possible that some astrophysical observables are influenced by the presence of such high temperatures, because of the modifications induced in the bulk and microscopic properties of dense matter. As an example, the gravitational wave spectrum generated in a neutron star merger might be influenced by the temperature dependence of the equation of state [2]. Also, the cooling of the neutron star after its birth is dominated by neutrino emission. This involves a series of processes that are particularly sensitive to the formation of Cooper pairs in the nuclear medium [3]. Consequently, astrophysical observations could help us constrain the temperature dependence of the microscopic and bulk properties of dense matter.

There is, however, another way to access the thermodynamical (TD) properties of nuclear and hadronic systems. In experimental facilities, "hot" nuclei are created in the collisions of heavy ions at intermediate energies [4]. There has been an increasing effort to interpret experimental data concerning these heavy-ion collisions in terms of the equation of state of nuclear matter [5,6]. More specifically, multifragmentation reactions at intermediate energies are used to access the properties of thermally equilibrated "blobs" of

*rios@nscl.msu.edu nuclear matter [7]. The evidence gathered in these experiments points toward the existence of a liquid-gas phase transition for nuclear systems at densities below the empirical saturation density, $\rho_{0}=0.16 \mathrm{fm}^{-3}$, and temperatures around $\sim 6-9 \mathrm{MeV}$ [8]. Empirically, the liquid-gas phase transition is usually discussed in terms of a plateau in the caloric curve for different types of reactions at different energies [9], although the presence of such a plateau may also be explained in terms of a density- and temperature-dependent effective mass [10]. Statistical models, which assume an equilibrated thermal freeze-out density, have had certain success in describing experimental results [11]. In any case, a common underlying idea in all these discussions is the assumption that some sort of thermal equilibrium is reached at a given stage of the multifragmentation reaction.

A first step toward a full theoretical understanding of the thermal properties of nuclear systems can be achieved by studying symmetric nuclear matter (an ideal, infinite, highdensity system composed of the same amount of neutrons and protons interacting via the strong force) at finite temperatures, because the features of the liquid-gas phase transition are in general easier to study in the homogeneous system. This is obviously a very crude approximation and can only be taken as a guide for a better theoretical understanding of the thermal properties of dense matter. As an example, the low-density phase of dense nuclear matter is not a homogeneous gas of nucleons and should instead be described in terms of droplets of light nuclei (deuterons, $\alpha$ particles). In the case of real experiments, moreover, finite size effects are as important as the bulk properties in determining the critical behavior. Finite nuclei can only be excited up to some limiting temperature above which Coulomb effects together with the decrease in surface tension lead to their thermal dissociation $[12,13]$. All in all, it is not an easy task to link the properties of the phase transition in the homogeneous case to those 
of finite nuclei [14-16]. In this paper, however, we shall concentrate on studying the liquid-gas phase transition in the ideal case of nuclear matter. Our basic goal is to discuss to what extent the properties of this transition depend on the many-body description chosen as well as on the underlying $N N$ interaction.

Traditionally, the studies of nuclear systems at finite temperature have been performed using effective interactions within mean-field theories, either relativistic $[17,18]$ or nonrelativistic [19,20]. The temperature dependence in these approaches arises mainly from the modification of the zero temperature step-like momentum distributions, which become Fermi-Dirac distributions. As a consequence, the mean-field and the bulk properties have a rather simple temperature dependence. Thermal effects on the correlations between strongly interacting nucleons are completely ignored. The effective interactions, which are fitted to describe the bulk properties of nuclei at zero temperature and thereby account for correlation effects in a phenomenological way, have no temperature dependence. This is in striking contrast with the more microscopically founded many-body calculations [21-23], where the in-medium interaction is found by using some sort of Pauli blocking. Such blocking effects are weakened by temperature, therefore giving rise to nontrivial temperature dependences in the microscopic and macroscopic properties of dense matter.

There are only a few realistic many-body calculations for nuclear matter at finite temperature [24-26]. Some of these have consistency problems, since they are not truly based on finite-temperature many-body theory, but on naive extensions of zero-temperature approaches to the nonzero-temperature domain. The Green's function approach is, however, based on the perturbative expansion of the single-particle propagator at finite temperature [27], and therefore it is a well-grounded approach that allows systematic improvement. In contrast, the variational approach is based on an explicit incorporation of the two-body nucleon-nucleon $(N N)$ correlations in the nuclear wave function. Its extension to finite temperatures has traditionally relied on the "frozen" correlation approximation; i.e., the correlation functions have been assumed to be the same at zero and at finite temperature [24,28,29]. This might be adequate for the high-density low-temperature phase, but it remains to be seen if it offers an appropriate description of the high-temperature phase. Recently there has been a substantial effort to discuss the more formal aspects associated with the variational approach at finite temperatures [30]. Let us also note that the variational approach is restricted by nature to deal with local potentials, while the self-consistent Green's function (SCGF) approach can in principle deal with any sort of realistic two-body force. The Brueckner-Hartree-Fock (BHF) approach has also been used in the study of hot nuclear systems [25], although the Brueckner-Bethe-Goldstone expansion, on which the BHF approach is based, is only valid at zero temperature. The standard finite-temperature generalization of this approach is, in a way, phenomenological and relies on the replacement of all the step-function momentum distributions of the zero temperature case with Fermi-Dirac ones. This finite-temperature extension is, however, not well defined at a fundamental level, since it does not take into account the contributions of anomalous diagrams [31]. A consistent Brueckner-like approach at finite temperature is given by the Bloch-de Dominicis formalism [32-34], which has only recently been applied to the nuclear many-body problem at finite temperatures by Baldo and coworkers [13,22]. Relativistic effects have also been explored in the framework of an extension to finite temperature of the Dirac-BruecknerHartree-Fock approach [35,36].

A good alternative for a suitable microscopic many-body description of hot correlated systems is provided by the perturbation expansion of the one-body Green's functions [37], which relies on the generalization of the Wick theorem at finite temperature [38]. Because of the strong short-range repulsion of the $N N$ interaction, the minimum meaningful scheme that can describe nuclear matter is provided by the ladder approximation. This arises from a decoupling of the three-body Green's function in terms of one- and two-body propagators [38] and can be cast as a set of self-consistent equations that describe the in-medium modifications of the nucleon due to the presence of the surrounding nucleons. These equations lead to an approximation that goes beyond the mean-field and quasiparticle pictures; i.e., the off-shell effects and the fragmentation of single-particle states are fully taken into account. This self-consistent Green's function (SCGF) approach is well established in nuclear physics [39] and has already been applied to studying the microscopic properties of nuclear matter at finite temperatures [40-43]. A major motivation for these studies has been the fact that, at nonzero temperature, one can avoid the numerical and physical problems associated with the neutron-proton pairing instability [44-46]. The SCGF, however, can also be used to study the TD properties of the system in the normal phase, by making use of the Luttinger-Ward (LW) formalism. This approach leads to thermodynamically consistent results, once the effects of correlations in the entropy have been carefully taken into account $[26,47,48]$. In the following, we shall use this formalism to study the properties of the liquid-gas phase transition in nuclear matter. Note, however, that the effect of three-body forces is not included in our approach, and, as a consequence, the saturation properties of nuclear matter are not well reproduced. The lack of three-body forces will also have an impact on the liquid branch of the phase transition, thus modifying to a certain extent our predictions for the critical properties of nuclear matter. Our results should be considered as a theoretical study quantifying the importance of short-range correlations on the liquid-gas phase transition.

In the next section, we briefly summarize the SCGF approach at finite temperature, and we discuss under which approximations the standard $\mathrm{BHF}$ at finite temperatures can be obtained from it. The third section will deal with the application of the LW formalism to the calculations of the TD properties of a correlated system of nucleons. The results for the liquid-gas phase transition will be discussed in the fourth section. Finally, a brief summary will be given in Sec. V.

\section{SCGF AT FINITE TEMPERATURE}

The key quantity in many-body Green's function theory is the single-particle propagator, which, in the grand-canonical 
ensemble, is defined according to

$$
i \mathcal{G}\left(\mathbf{k} t, \mathbf{k}^{\prime} t^{\prime}\right)=\operatorname{Tr}\left\{\hat{\rho} \mathcal{T}\left[a_{\mathbf{k}}(t) a_{\mathbf{k}^{\prime}}^{\dagger}\left(t^{\prime}\right)\right]\right\},
$$

where we have introduced the density matrix operator

$$
\hat{\rho}=\frac{1}{Z} e^{-\beta(\hat{H}-\mu \hat{N})},
$$

and the partition function

$$
Z=\operatorname{Tr}\left\{e^{-\beta(\hat{H}-\mu \hat{N})}\right\} .
$$

In the previous equations, $\beta=1 / T$ denotes the inverse temperature, and $\mu$ is the chemical potential. $\mathcal{T}$ stands for a time-ordering operator, and the traces $\operatorname{Tr}\{\cdot\}$ are to be taken over all energy and particle number eigenstates. One can express the single-particle propagator in Fourier-energy space by means of the spectral decomposition

$$
\begin{aligned}
\mathcal{G}(k, \omega)= & \int_{-\infty}^{\infty} \frac{d \omega^{\prime}}{2 \pi} \frac{A^{>}\left(k, \omega^{\prime}\right)}{\omega-\omega^{\prime}+i \eta} \\
& +\int_{-\infty}^{\infty} \frac{d \omega^{\prime}}{2 \pi} \frac{A^{<}\left(k, \omega^{\prime}\right)}{\omega-\omega^{\prime}-i \eta}
\end{aligned}
$$

where the finite-temperature equivalent of the $T=0$ hole spectral function is given by the Lehmann representation

$$
\begin{aligned}
A^{<}(k, \omega)= & 2 \pi \sum_{n, m} \frac{e^{-\beta\left(E_{m}-\mu N_{m}\right)}}{Z}\left|\left\langle\Psi_{n}\left|a_{k}\right| \Psi_{m}\right\rangle\right|^{2} \\
& \times \delta\left[\omega-\left(E_{m}-E_{n}\right)\right] .
\end{aligned}
$$

The main difference with respect to the zero-temperature case comes from the average over the thermal bath in the initial states. A similar definition holds for $A^{>}(k, \omega)$, with the replacement $a_{k} \rightarrow a_{k}^{\dagger}$. In thermal equilibrium, both spectral functions are related by the Kubo-Martin-Schwinger relation

$$
A^{>}(k, \omega)=e^{\beta(\omega-\mu)} A^{<}(k, \omega) .
$$

In contrast to the zero-temperature case, the energy domains of $A^{<}(k, \omega)$ and $A^{>}(k, \omega)$ are not separated by the Fermi energy, and both spectral functions are defined for all energies. The total spectral function $A(k, \omega)$ is given by the sum of the two functions $A^{<}$and $A^{>}$, and therefore it can be expressed in terms of the values of $\mathcal{G}$ close to the real axis as

$$
A(k, \omega)=-2 \operatorname{ImG}\left(k, \omega_{+}\right),
$$

where we have introduced the notation $\omega_{+}=\omega+i \eta$. Since the spectral function completely determines the one-body propagator via the previous equation, all the one-body properties of the system can be expressed in terms of it.

In the medium, the single-particle Green's function can be obtained from Dyson's equation:

$$
\left[\omega-\frac{\hbar^{2} k^{2}}{2 m}-\Sigma(k, \omega)\right] \mathcal{G}(k, \omega)=1,
$$

where $\Sigma(k, \omega)$ denotes a complex self-energy. The self-energy accounts for the interactions of a particle with the other particles in the medium. It fulfills the following spectral decomposition:

$$
\Sigma(k, z)=\Sigma^{\mathrm{HF}}(k)-\int \frac{d \omega}{2 \pi} \frac{2 \operatorname{Im} \Sigma\left(k, \omega_{+}\right)}{z-\omega},
$$

where $z$ is a complex variable, and the term $\Sigma^{\mathrm{HF}}(k)$ is a real energy-independent generalized Hartree-Fock contribution

$$
\Sigma^{\mathrm{HF}}(k)=\int \frac{d^{3} k^{\prime}}{(2 \pi)^{3}}\left\langle\mathbf{k} \mathbf{k}^{\prime}|V| \mathbf{k} \mathbf{k}^{\prime}\right\rangle_{A} n\left(k^{\prime}\right),
$$

with $n(k)$ being the single-particle momentum distribution

$$
n(k)=\int \frac{d \omega}{2 \pi} A(k, \omega) f(\omega),
$$

and where $f(\omega)=\left[e^{\beta(\omega-\mu)}+1\right]^{-1}$ stands for the Fermi-Dirac distribution. The imaginary part of the self-energy, necessary to compute the second term in Eq. (9), is obtained by letting $z \rightarrow \omega_{+}$in Eq. (9), and it is related to the effective twobody $N N$ interaction in the medium (the so-called scattering $T$ matrix):

$$
\begin{aligned}
\operatorname{Im} & \Sigma\left(k, \omega_{+}\right) \\
= & \int \frac{d^{3} k^{\prime}}{(2 \pi)^{3}} \int_{-\infty}^{\infty} \frac{d \omega^{\prime}}{2 \pi}\left\langle\mathbf{k} \mathbf{k}^{\prime}\left|\operatorname{Im} T\left(\omega+\omega_{+}^{\prime}\right)\right| \mathbf{k} \mathbf{k}^{\prime}\right\rangle_{A} \\
& \times A\left(k^{\prime}, \omega^{\prime}\right)\left[f\left(\omega^{\prime}\right)+b\left(\omega+\omega^{\prime}\right)\right] .
\end{aligned}
$$

Note the presence of a Bose-Einstein distribution, $b(\Omega)=$ $\left[e^{\beta[\Omega-2 \mu]}-1\right]^{-1}$, as a consequence of the symmetric treatment of particle-particle and hole-hole correlations.

The effective in-medium interaction is calculated in the ladder approximation. This accounts for the repeated scattering of particles in the medium, and it is well suited for the lowdensity strong-interaction regime of interest for nuclear matter [27]. The $T$ matrix is determined by the solution of the integral equation

$$
\begin{aligned}
& \left\langle\mathbf{k} \mathbf{k}^{\prime}\left|T\left(\Omega_{+}\right)\right| \mathbf{p} \mathbf{p}^{\prime}\right\rangle_{A} \\
& =\left\langle\mathbf{k k}^{\prime}|V| \mathbf{p} \mathbf{p}^{\prime}\right\rangle_{A}+\int \frac{d^{3} q}{(2 \pi)^{3}} \int \frac{d^{3} q^{\prime}}{(2 \pi)^{3}}\left\langle\mathbf{k} \mathbf{k}^{\prime}|V| \mathbf{q} \mathbf{q}^{\prime}\right\rangle_{A} \\
& \quad \times \mathcal{G}_{\mathrm{II}}^{0}\left(\mathbf{q}, \mathbf{q}^{\prime}, \Omega_{+}\right)\left\langle\mathbf{q q} \mathbf{q}^{\prime}\left|T\left(\Omega_{+}\right)\right| \mathbf{p} \mathbf{p}^{\prime}\right\rangle_{A},
\end{aligned}
$$

where the intermediate propagator accounts for the propagation of two noninteracting but dressed nucleons:

$$
\begin{aligned}
\mathcal{G}_{\mathrm{II}}^{0}\left(k_{1}, k_{2}, \Omega_{+}\right)= & \int_{-\infty}^{\infty} \frac{d \omega}{2 \pi} \int_{-\infty}^{\infty} \frac{d \omega^{\prime}}{2 \pi} A\left(k_{1}, \omega\right) A\left(k_{2}, \omega^{\prime}\right) \\
& \times \frac{1-f(\omega)-f\left(\omega^{\prime}\right)}{\Omega_{+}-\omega-\omega^{\prime}} .
\end{aligned}
$$

To reduce the dimensionality of Eq. (13), one usually relies in the standard partial-wave decomposition. An extra simplification is achieved by using an angle average of the two-body propagator with respect to the center of mass and relative momentum of the two colliding particles [48,49].

Equations (9)-(14) form a closed set of equations that can be solved self-consistently. In terms of numerics, it is advantageous to work at constant density, and therefore we supplement the previous set of equations with the normalization of the momentum distribution:

$$
\rho=v \int \frac{d^{3} k}{(2 \pi)^{3}} n(k),
$$

where $v$ denotes the degeneracy of the system $(v=4$ in the case of symmetric nuclear matter). Once convergence 
is reached in the self-consistent procedure for a given temperature and density, one has access to the spectral function $A(k, \omega)$, which, loosely speaking, describes the probability of finding a nucleon in the medium with momentum $k$ and energy $\omega$. At this point, one can calculate several microscopic and macroscopic properties of the system. The momentum distribution, for instance, can be computed using Eq. (11). The energy per particle is also accessible from the GalitskiiMigdal-Koltun sum rule:

$$
\begin{aligned}
\frac{E}{A}(\rho, T)= & \frac{v}{\rho} \int \frac{d^{3} k}{(2 \pi)^{3}} \int_{-\infty}^{\infty} \frac{d \omega}{2 \pi} \frac{1}{2}\left(\frac{k^{2}}{2 m}+\omega\right) \\
& \times A(k, \omega) f(\omega),
\end{aligned}
$$

which is valid for a Hamiltonian with only two-body interactions. The importance of self-consistency in the calculations stems from the fact that it immediately leads to the conservation of both microscopic and macroscopic properties [50]. In addition, it guarantees the fulfillment of the sum rules for the one-body spectral function [51,52].

In this paper, we shall make comparisons between the finite-temperature SCGF and BHF approaches. The latter can be formally derived from the first by performing some particular approximations. First, one has to assume that, for a given momentum, all the strength of the spectral function is accumulated in one energy, $A(k, \omega)=\delta\left[\omega-\varepsilon^{\mathrm{BHF}}(k)\right]$, with $\varepsilon^{\mathrm{BHF}}(k)$ the BHF single-particle energy. This simplifies the calculation of the noninteracting two-body propagator of Eq. (14), which becomes a finite-temperature Pauli blocking factor involving both particle-particle and hole-hole propagation. Since in the BHF approach only intermediate particleparticle states are considered, the phase-space factor needs to be properly modified, $1-f(\omega)-f\left(\omega^{\prime}\right) \rightarrow[1-f(\omega)][1-$ $f\left(\omega^{\prime}\right)$ ]. Finally, in the BHF self-energy, one does not take into account the contribution of the Bose function in Eq. (12). After the BHF equations are iterated and consistency is reached, one obtains a single-particle spectrum and an in-medium $G$-matrix interaction, the real part of which is used to obtain the energy per particle of the system:

$$
\begin{aligned}
\frac{E}{A}(\rho, T)= & \frac{v}{\rho} \int \frac{d^{3} k}{(2 \pi)^{3}} \frac{k^{2}}{2 m} f\left[\varepsilon^{\mathrm{BHF}}(k)\right]+\frac{v}{2 \rho} \int \frac{d^{3} k}{(2 \pi)^{3}} \frac{d^{3} k^{\prime}}{(2 \pi)^{3}} \\
& \times\left\langle\mathbf{k k}^{\prime}\left|\operatorname{Re} G\left(\Omega=\varepsilon^{\mathrm{BHF}}(k)+\varepsilon^{\mathrm{BHF}}\left(k^{\prime}\right)_{+}\right)\right| \mathbf{k k}^{\prime}\right\rangle_{A} \\
& \times f\left[\varepsilon^{\mathrm{BHF}}(k)\right] f\left[\varepsilon^{\mathrm{BHF}}\left(k^{\prime}\right)\right] .
\end{aligned}
$$

The SCGF and the BHF approaches with two-body $N N$ interactions cannot reproduce the saturation properties of nuclear matter at zero temperature because of the lack of repulsive contributions, most probably those coming from three-body forces $[22,53]$. The region of interest for liquid-gas phase transition studies is, however, in the low-density regime (critical densities are typically $\frac{1}{2}$ to $\frac{1}{3}$ of $\rho_{0}$ ), and this density regime should not be strongly modified by the presence of three-body forces. Estimates of the importance of the threebody forces in the liquid-gas regime have been performed within the BHF approximation, indicating small modifications to the critical properties [22,53].

To assess the model dependence of the properties of this transition, we will compare the results obtained with two different $N N$ interactions: the CDBONN [54] and the Argonne V18 [55] potentials. Although both of them reproduce the scattering phase shifts up to about $300 \mathrm{MeV}$, they have very different short-range cores, off-shell structure, and tensor components. The many-body calculations depend on these details; therefore the properties of dense matter, in particular the critical properties of the liquid-gas phase transition, will be different for the two interactions. To our knowledge, this is the first time that two different realistic interactions are used within the SCGF approach to study the liquid-gas coexistence and the critical properties. In all the calculations quoted in the following and for both the SCGF and the BHF approximations, partial waves up to $J=8$ have been included. The in-medium effective interactions have been computed with $J \leqslant 4$, and the Born approximation has been used for $J>4$.

\section{THERMODYNAMICAL PROPERTIES OF NUCLEAR MATTER}

A complete TD description of the system requires the computation of the free energy, $F=E-T S$. As indicated in the previous section, the internal energy in the SCGF approach can be calculated from the one-body propagator via the Galitskii-Migdal-Koltun sum rule. Therefore, a suitable method for the calculation of the entropy is required to describe the thermodynamics of the system. The LW formalism can be used to find an expression of the grand-canonical potential $\Omega$ in terms of dressed propagators [31,56,57]. Because this expression of $\Omega$ is stationary with respect to variations of the one-body Green's function, one can easily compute the entropy from the derivative $S=-\left.\frac{\partial \Omega}{\partial T}\right|_{\mu}$. This entropy can be split into two terms: $S=S^{\mathrm{DQ}}+S^{\prime}$. The first one is the so-called dynamical quasiparticle (DQ) entropy density

$$
S^{\mathrm{DQ}}=v \int \frac{d^{3} k}{(2 \pi)^{3}} \int_{-\infty}^{\infty} \frac{d \omega}{2 \pi} \sigma(\omega) B(k, \omega),
$$

defined as the convolution of a statistical factor $\sigma(\omega)=$ $-f(\omega) \ln f(\omega)-[1-f(\omega)] \ln [1-f(\omega)]$ and a spectral function $B(k, \omega)$, which is related to the single-particle spectral function $A(k, \omega)$ and the self-energy by the following equation:

$$
\begin{aligned}
B(k, \omega)= & A(k, \omega)\left[1-\frac{\partial \operatorname{Re} \Sigma(k, \omega)}{\partial \omega}\right] \\
& -2 \frac{\partial \operatorname{Re} \mathcal{G}(k, \omega)}{\partial \omega} \operatorname{Im} \Sigma\left(k, \omega_{+}\right) .
\end{aligned}
$$

This expression takes into account the correlations of the dressed particles in the medium, since it includes finite width effects. In this paper, we shall make the assumption that $S^{\prime}$ is negligible. As a matter of fact, Carneiro and Pethick have shown that its effects are constrained by phasespace restrictions [57]. This assumption is also confirmed by the fact that the results ignoring the contribution of $S^{\prime}$ are thermodynamically consistent [47], as we shall see in the following. The free energy will be computed from now on from the difference between the Galitskii-Migdal-Koltun sum-rule energy and the DQ entropy, $F=E^{\mathrm{GMK}}-T S^{\mathrm{DQ}}$. In the BHF approach, the entropy should not include any effect due to the 


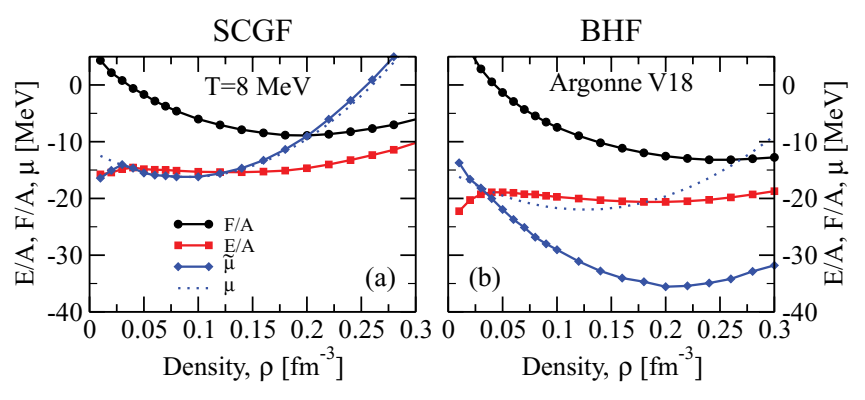

FIG. 1. (Color online) Energy per particle (circles), free energy per particle (squares) and microscopic chemical potentials $\tilde{\mu}$ (diamonds) for (a) the SCGF approach and (b) the BHF approach, as a function of the density at $T=8 \mathrm{MeV}$ for the Argonne V18 potential. The macroscopic chemical potential $\mu$ is also shown (dotted line).

widening of the quasi-particle peak and therefore it will be computed from the mean-field expression:

$$
S^{\mathrm{BHF}}=v \int \frac{d^{3} k}{(2 \pi)^{3}} \sigma\left[\varepsilon^{\mathrm{BHF}}(k)\right] .
$$

The free energy per particle, together with the energy per particle and the chemical potential, is shown in Figs. 1 and 2 as a function of the density at constant temperature. In Fig. 1, we display the results at $T=8 \mathrm{MeV}$ for the Argonne V18 interaction, while in Fig. 2 we consider the CDBONN interaction at $T=10 \mathrm{MeV}$. Both temperatures are sufficiently below the corresponding critical temperatures, as we will see. Note that in both figures panel (a) corresponds to the SCGF results and panel (b) to the BHF ones. The latter have been obtained from the combination of Eq. (17) for the energy and Eq. (20) for the entropy. Let us first discuss the differences between the two many-body approaches. It is already well-established that hole-hole propagation, which is included in the SCGF but not in the BHF approach, yields a repulsive contribution to the energy per particle of about 4-6 MeV close to saturation density [41,58]. Since this repulsive contribution tends to increase with the nuclear density, one obtains a smaller saturation density in the SCGF than in the BHF approach. A similar effect is also observed for the free energy per particle, since both the dynamical quasiparticle and the BHF entropies are quite close to each other [47].

The temperatures considered in Figs. 1 and 2 are slightly different from each other, so one should be cautious when

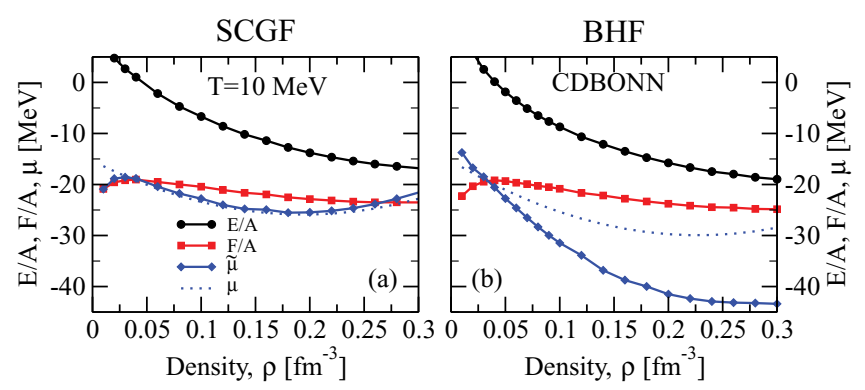

FIG. 2. (Color online) Same as Fig. 1, but for the CDBONN potential at $T=10 \mathrm{MeV}$. comparing these results. Nevertheless, this comparison exhibits the main features that have frequently been discussed in the literature for the energy as a function of density at zero temperature: the CDBONN interaction contains weaker tensor components than the Argonne V18. This implies that the density-dependent suppression effects in the iterated tensor terms are less efficient for the former interaction than for the latter. This leads to a more attractive energy per nucleon and a larger saturation density for the CDBONN than for Argonne V18 interaction, features which are also present in Figs. 1 and 2.

It is interesting to compare the free energy and the chemical potential to check the fulfillment of TD consistency. Some properties of the system can be computed either microscopically (from Green's function theory) or macroscopically (from the TD properties of the system). A TD consistent many-body approximation will yield the same result for both of them. A very sensitive quantity to this test is the chemical potential. On the one hand, it can be computed microscopically from the normalization of the momentum distribution, Eq. (15), giving rise to the microscopic chemical potential $\tilde{\mu}$ (diamonds in Figs. 1 and 2). On the other hand, one can compute it from the derivative of the free energy with respect to the number of nucleons at constant temperature, $\mu=\frac{\partial F}{\partial N}$ (dotted lines). The differences between $\tilde{\mu}$ and $\mu$ for the BHF approach can be larger than $15 \mathrm{MeV}$, showing its lack of consistency. Note that, in particular, the Hugenholtz-van Hove theorem is violated, i.e., $\tilde{\mu}$ does not coincide with $\frac{F}{A}$ at its minimum. The violation seems to be larger for CDBONN, $\sim 20 \mathrm{MeV}$, than for Argonne V18, $\sim 10 \mathrm{MeV}$. The SCGF results, however, fulfill TD consistency with less than $1 \mathrm{MeV}$ error in a wide density range for both interactions. Note that $\mu$ has been computed by fitting a fourth-order polynomial to the free-energy density and determining the derivatives from this polynomial fit. The lack of accuracy in the fit is responsible for the deviations at very low densities. In any case, the numerical implementation of the ladder approximation by means of the SCGF scheme leads to TD consistent results, independently of the $N N$ potential under consideration [59].

The pressure is shown as a function of density in Fig. 3 for several temperatures and for both the SCGF and BHF approaches. Figures 3(a) and 3(b) correspond to Argonne V18; and Figs. 3(c) and 3(d) to CDBONN. The pressure is obtained from the TD relation, $p=\rho(\mu-F / A)$. Because of the conserving properties of the SCGF approach, we can compute the pressure at every density and temperature by using the microscopic chemical potential in the previous expression. For BHF, however, $\mu$ has to be computed as a numerical derivative of the free energy with respect to $\rho$. Note that numerical problems can appear in the low density limit as a result of the logarithmic density dependences in this region.

In general, one can say that the repulsive effect of the holehole propagation in the free energy, which we have already discussed above, is translated into larger pressures in the SCGF than in the BHF approach, especially at large densities. The SCGF method, therefore, yields a stiffer equation of state than the BHF approach. By construction, the TD chemical potential $\mu$ crosses the free-energy curve at its minimum, thus yielding a point of zero pressure. This defines the saturation 


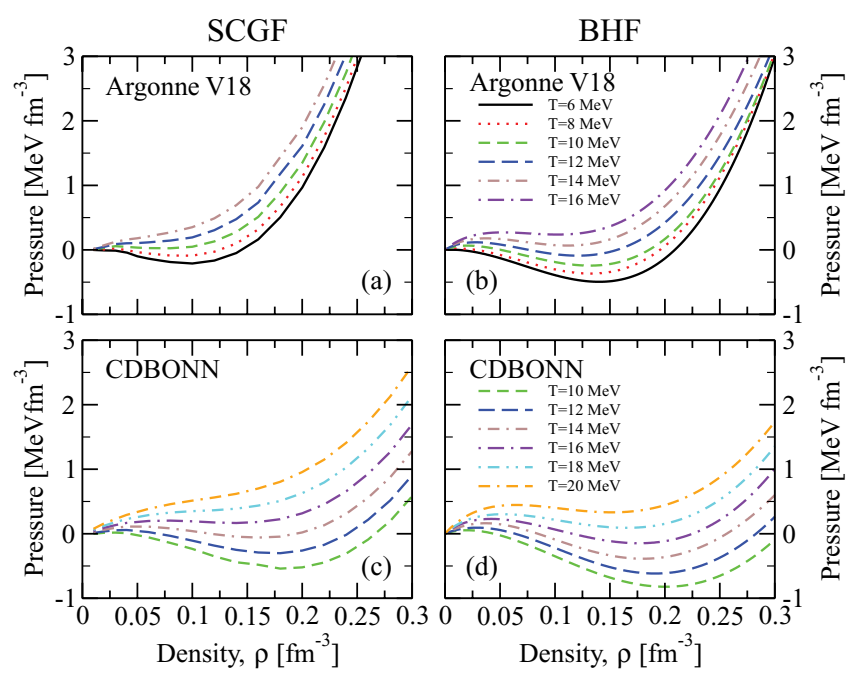

FIG. 3. (Color online) Pressure as a function of density for several temperatures, obtained from SCGF and BHF calculations with the Argonne V18 and CDBONN interactions.

density at each temperature. The repulsive effect induced by the propagation of holes in the SCGF approach is reflected in a smaller saturation density with respect to BHF, i.e., the minimum of the free energy is shifted to smaller densities in the SCGF approach. This effect has already been observed at zero temperature [58], and it appears to hold when thermal effects are taken into account. Above a certain temperature, the minimum of the free energy per particle disappears, and the equation $P(\rho)=0$ no longer has a solution. This defines the so-called flashing temperature $T_{f}$. Table I gives the flashing temperature for the different approaches and potentials. The SCGF results lead to $T_{f}$ values that are about $3 \mathrm{MeV}$ lower than the BHF ones.

The differences in pressure from the two many-body approaches are sizable, but the differences due to the change of potential are even larger. The pressure for Argonne V18, for instance, increases much more steeply with density than that for CDBONN. There are also substantial differences in the saturation densities induced by the two potentials at all temperatures, with Argonne V18 leading to lower saturation densities than CDBONN. This is in agreement with the fact that at $T=0$ the BHF saturation density is much larger for CDBONN than for Argonne V18 [60]. Note also that the temperature ranges explored in the upper and lower panels of Fig. 3 are not the same. The temperature dependences induced by the two potentials are therefore rather different. For instance, the flashing temperatures for Argonne V18 are about 4-5 MeV lower than for CDBONN with both SCGF
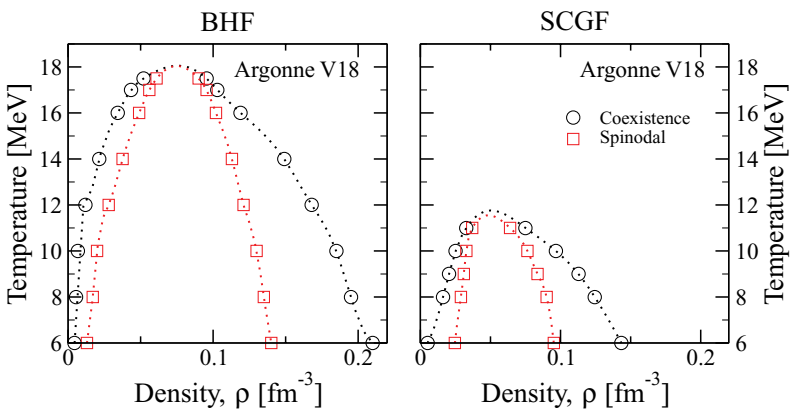

FIG. 4. (Color online) Coexistence and spinodal lines for symmetric nuclear matter within the BHF and SCGF approaches for the Argonne V18 interaction.

and BHF. This suggests that the different off-shell and tensor components of the $N N$ forces affect not only the properties of nuclear matter at zero temperature but also its TD properties in an important way. It remains to be seen if the experimental knowledge gathered about the thermodynamical properties of nuclear systems can provide additional information to constrain the $N N$ interaction and select the proper many-body approach to be used in their description.

For both approaches and potentials, the pressure decreases with density in a given range. This signals the existence of a mechanical instability, which is associated with a first-order liquid-gas phase transition. The properties of this transition are studied in the following section.

\section{LIQUID-GAS PHASE TRANSITION}

A physical interpretation of the TD unstable zone is customarily obtained by making use of the Maxwell construction. For each temperature, one should find the gas and liquid density, for which the equations $\mu\left(\rho_{g}\right)=\mu\left(\rho_{l}\right)$ and $p\left(\rho_{g}\right)=p\left(\rho_{l}\right)$ are simultaneously satisfied. For a given temperature, the range $\rho_{g}-\rho_{l}$ gives the coexistence region, where the gas and liquid phases coexist at constant pressure and chemical potentials. The spinodal region is defined by the violation of the TD stability criteria, $\frac{\partial \mu}{\partial \rho}>0$ and $\frac{\partial p}{\partial \rho}>0$. For a one-component system, both conditions are equivalent. This spinodal region lies within the liquid-gas coexistence region in the densitytemperature plane, and the region between the two curves defines the so-called metastable region. Finding the spinodal and coexistence densities at each temperature, one obtains the phase diagrams shown in Fig. 4 for Argonne V18 and in Fig. 5 for CDBONN. Note that the two figures have different vertical scales because of the large differences in the TD

TABLE I. Critical properties for different approximations and $N N$ interactions.

\begin{tabular}{lcccccc}
\hline \hline Potential & Approach & $T_{f}(\mathrm{MeV})$ & $T_{c}(\mathrm{MeV})$ & $\rho_{c}\left(\mathrm{fm}^{-3}\right)$ & $p_{c}\left(\mathrm{MeV} \mathrm{fm}^{-3}\right)$ & $\frac{p_{c}}{T_{c} \rho_{c}}$ \\
\hline Argonne V18 & SCGF & 9.5 & 11.6 & 0.05 & 0.08 & 0.14 \\
& BHF & 13.1 & 18.1 & 0.08 & 0.40 & 0.28 \\
CDBONN & SCGF & 14.4 & 18.5 & 0.11 & 0.40 & 0.20 \\
& BHF & 17.2 & 23.3 & 0.11 & 0.73 & 0.28 \\
\hline \hline
\end{tabular}



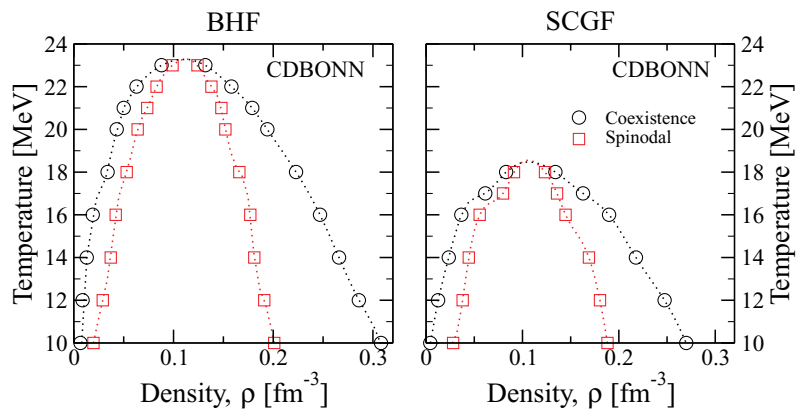

FIG. 5. (Color online) Same as Fig. 4, but for the CDBONN interaction.

properties induced by the two interactions. Once again, let us emphasize that one should be cautious when using fitting procedures for the free energy. These are necessary to obtain suitable analytical expressions for the derivatives of $F(\rho, T)$, needed to implement the liquid-gas coexistence conditions. It is also worth noting that for the temperatures considered, both methods (BHF and SCGF) are numerically stable in a range of densities from very low ones (gas phase, $\rho=0.01 \mathrm{fm}^{-3}$ ) to relatively large ones (liquid phase, $\rho=0.30 \mathrm{fm}^{-3}$ ). For the SCGF approach, numerical difficulties related to the pairing instability appear at lower temperatures [44,45].

The critical temperature for the liquid-gas phase transition of symmetric nuclear matter corresponds to the maximum of the spinodal and coexistence lines, which coincide with each other at the critical point. The flashing temperature $T_{f}$ always lies below $T_{c}$ and, as noted above, corresponds to the maximum temperature at which the pressure presents a node. $T_{f}$ represents the highest temperature that finite nuclei can withstand without thermally dissociating. The critical properties as well as the limiting temperatures for the two approaches and interactions are listed in Table I. Both $T_{f}$ and $T_{c}$ depend strongly on the type of approximation used to study nuclear matter and also on the $N N$ interaction. Note that for a given potential, BHF results always lead to larger flashing and critical temperatures (about 3-4 MeV larger) than SCGF. Also, the results for Argonne V18 are, within each approximation, about 4-5 MeV lower than those of CDBONN.

At this point, we are going to compare our predictions for the critical temperature with those obtained in other approximation schemes. We obtain the largest critical temperature for the CDBONN potential in the BHF approximation, with a value of $T_{c}=23.3 \mathrm{MeV}$. The critical temperature for CDBONN using the SCGF approach is, however, $T_{c}=$ $18.5 \mathrm{MeV}$. This is in close agreement with the value of $T_{c}=$ $18 \mathrm{MeV}$ obtained with the somewhat similar Bonn B potential in the Bloch-de Dominicis formalism [13]. The Argonne V18 interaction yields lower critical temperatures when compared to CDBONN, with $T_{c}=18.1 \mathrm{MeV}$ for $\mathrm{BHF}$ and $T_{c}=$ 11.6 MeV for SCGF. The first result can be compared with calculations performed with other approximations and the same $N N$ potential. The LOCV results of Ref. [28] correspond to a somewhat larger critical temperature of $T_{c}=22.2 \mathrm{MeV}$ for V18, while a more recent variational calculation with frozen correlations and three-body forces leads to $T_{c}=$
$18 \mathrm{MeV}$ [29], rather close to our BHF result. The authors of Ref. [53] found a critical temperature $T_{c}=16 \mathrm{MeV}$ when the effect of three-body forces was neglected. Other results in the range 16-20 MeV have been obtained with similar interactions. One can estimate the critical temperature of the variational calculation by Friedman and Pandharipande with the Urbana V14 potential (plus three-body force) to be about $T_{c}=17-18 \mathrm{MeV}$ [24]. The Bloch-de Dominicis calculation of Ref. [22] for the Argonne V14 interaction yields $T_{c}=$ $21 \mathrm{MeV}$ with two-body forces and $T_{c}=20 \mathrm{MeV}$ when the effect of three-body forces is included. In all these cases, the critical temperature is about 7-9 MeV larger than the one obtained with Argonne V18 in the SCGF approach. This is in fact below the usually quoted critical temperature for infinite matter, of around 15-20 MeV. Yet, some other models, especially the relativistic ones, have found similar low values of $T_{c}$. The density-dependent relativistic mean-field calculation of Ref. [18] found critical temperatures of the order of $12 \mathrm{MeV}$. The Dirac-Brueckner-Hartree-Fock calculations also yield low critical temperatures, such as the $T_{c}=12$ and $T_{c}=10.4 \mathrm{MeV}$ of Refs. [35] and [36], respectively, obtained using one-boson-exchange interactions fitted to $N N$ data. The nonrelativistic semirealistic model of Ref. [61] found convergence problems, possibly associated with the liquid-gas transition, below a critical temperature as low as $9 \mathrm{MeV}$.

Can one explain in simple terms the origin of the large differences in critical temperatures between the two approaches and $N N$ interactions? In fact, there are some simple models that try to relate the critical properties to the saturation properties of nuclear matter at zero temperature [14,62]. A particularly useful and simple estimate is obtained from the Kapusta model [15], which supposes that the temperature dependences are quadratic (as in the free Fermi gas close to the degenerate limit) and modulated by an effective mass $m^{*}$ which governs the density of states. Under the additional assumption that the zero-temperature energy per particle can be characterized by the compressibility $K$, one finds that

$$
T_{c}=0.326\left(\frac{K}{m^{*}}\right)^{1 / 2} \rho_{0}^{1 / 3},
$$

i.e., the critical temperature increases with $K$ and the saturation density $\rho_{0}$. The presence of the pairing instability $[44,45]$ prevents us from decreasing the temperature in the SCGF scheme below about $5 \mathrm{MeV}$, and we cannot safely extrapolate the values of $K, m^{*}$, and $\rho_{0}$ to the zero-temperature limit. We have, however, performed BHF calculations at zero temperature and found the compressibility $K=279 \mathrm{MeV}(K=212 \mathrm{MeV})$ and the saturation density $\rho_{0}=0.35 \mathrm{fm}^{-3}\left(\rho=0.23 \mathrm{fm}^{-3}\right)$ for CDBONN (Argonne V18). The estimates obtained with Eq. (21) with a free mass lead to the critical temperatures $T_{c} \sim 25$ and $T_{c} \sim 19 \mathrm{MeV}$, in rather good agreement with the values reported in Table I. The available results of SCGF calculations seem to indicate that the saturation point decreases with respect to the BHF calculations; the compressibility, however, increases [58]. Within the Kapusta model, these two features would essentially compensate for each other and predict a critical temperature for the SCGF approach which is about the same as the one obtained in the BHF 
approximation. Note, however, that the SCGF approach leads to an enhancement of the density of states at low excitation energies which could be described in terms of a larger effective mass $m^{*}$ as compared to BHF [63].

Independent of this analysis, it is generally true that the critical temperature is correlated and somewhat close to the binding energy at saturation, $T_{c} \sim E / A$ [14]. The binding energies with $\mathrm{BHF}$ at zero temperatures are $E / A=21.6 \mathrm{MeV}$ for CDBONN and $E / A=16.2 \mathrm{MeV}$ for Argonne V18. Note that the 4-5 MeV difference in those values is close to the difference observed in the critical temperatures. Now, since usually the SCGF leads to binding energies that are 4-6 MeV more repulsive than those of the BHF approach, this simple argument would suggest that the critical temperatures should also decrease by a similar amount, as observed in Table I.

The Kapusta model also predicts the value of the critical density, $\frac{\rho_{c}}{\rho_{0}}=\frac{5}{12}=0.417$. For the BHF calculations, we find $\frac{\rho_{c}}{\rho_{0}}=0.35$ for Argonne V18 and $\frac{\rho_{c}}{\rho_{0}}=0.31$ for CDBONN, which are somewhat closer to the empirical formula $\frac{\rho_{c}}{\rho_{0}}=\frac{1}{3}$ [14]. Note also that the value for Argonne V18 in the BHF approximation lies within the range $0.07-0.09 \mathrm{fm}^{-3}$ quoted in Ref. [22] for the V14 potential. The spread in critical densities is significant when we change from one potential to another, but it does not differ so much when we consider different approximations. The differences are, however, more drastic for the critical pressure, which changes by almost an order of magnitude when comparing different approximations and potentials. Yet, surprisingly, the discrepancies are somewhat less important for the adimensional parameter $\frac{p_{c}}{\rho_{c} T_{c}}$. For a van der Waals equation of state, this parameter is $\frac{3}{8}=0.375$. The results of the last column of Table I are quite below this value. Intriguingly, the BHF results seem to lead to the same value, in spite of the large differences in each of their critical parameters.

Finally, let us recall again that we have not considered any three-body forces in the previous calculations. In terms of the phase diagram, one expects that the inclusion of three-body forces will shift the liquid coexistence branch to lower densities in the low-temperature phase but will presumably have a small effect at large temperatures. The effects on the gas phase, if any, would probably be very small in the homogeneous case. In fact, previous evaluations of the three-body effects on the critical temperature obtained within the BHF approach seem to indicate that these are rather small, about 1-3 MeV $[22,53]$. Such a decrease is much smaller than the discrepancies observed here when changing the two-body interaction or the many-body method.

\section{SUMMARY AND CONCLUSIONS}

We have calculated the TD properties of symmetric nuclear matter within the self-consistent Green's function and the Brueckner-Hartree-Fock approaches for two realistic $N N$ interactions, the CDBONN and the Argonne V18 potentials. The calculations cover a wide range of densities and temperatures. In the SCGF-LW approach, the entropy has been computed within the dynamical quasiparticle approximation, which takes into account the effects of correlations in the width of the quasiparticle peak. A very good agreement between the microscopic and macroscopic chemical potentials is found, highlighting the TD consistency of the SCGF-LW approach at the numerical level. This is in contrast to the BHF approximation, which yields a violation of the Hugenholtzvan Hove theorem by $10 \mathrm{MeV}(20 \mathrm{MeV})$ for the Argonne (CDBONN) potential.

The essential difference between SCGF and BHF is the consistent inclusion of hole-hole propagation terms in the former approach, leading to nontrivial spectral distribution functions and partial occupation probabilities for states with momenta above and below the Fermi momentum. These holehole terms tend to provide some repulsion, which increases with density. This feature is also reflected in the calculated pressure, for which the SCGF approach yields larger values, in particular at higher densities. This implies that the equation of state derived within the SCGF approach tends to be stiffer than the corresponding one evaluated within the BHF approximation. The repulsive effect of the hole-hole terms also leads to a lower flashing temperature for the SCGF than for the BHF approach.

When comparing the results between two different $N N$ interactions, we also find substantial differences, which are larger than those induced by the use of different many-body approaches. In particular, the Argonne V18 interaction leads to a stiffer equation of state and a lower flashing temperature than CDBONN.

The liquid-gas phase diagram for nuclear matter has been studied for the first time in the framework of the microscopic SCGF approach for two realistic $N N$ interactions and critically compared with the results obtained in the BHF approach. Substantial differences for the critical properties are found when changing the potential and the many-body approximation. The SCGF leads to critical temperatures that are 5-7 MeV lower than those obtained with the BHF approximation. Within the same approximation, CDBONN leads to results that are 6-7 MeV larger. For BHF, where $T=0$ calculations can be performed safely, we find that the critical density is about a third of the saturation density and that the critical temperature can be well approximated by Eq. (21), in terms of the compressibility and the saturation density.

Of course, to have a proper estimation of the critical temperature for finite nuclei, one should also take into account Coulomb effects and the existence of a surface tension. These results would further reduce the critical temperature, by a factor of $1 / 2-1 / 4[9,13]$. In this paper, we have found that realistic calculations allow for a large range of critical temperatures, in the same way that they predict different saturation properties. In particular, the important reduction in critical temperatures found for the Argonne interaction might be relevant when trying to connect the data of multifragmentation reactions with the liquid-gas phase transition for bulk nuclear matter.

\section{ACKNOWLEDGMENTS}

The authors are grateful to Isaac Vidana for useful and stimulating discussions and for the use of his BHF codes. 
This work was partially supported by the NSF under Grant No. PHY-0555893 and by the MEC (Spain) and FEDER under
Grant No. FIS2005-03142 and 2005SGR-00343 (Generalitat de Catalunya).
[1] M. Prakash, I. Bombaci, M. Prakash, P. J. Ellis, J. M. Lattimer, and R. Knorren, Phys. Rep. 280, 1 (1996).

[2] R. Oechslin and H.-T. Janka, Phys. Rev. Lett. 99, 121102 (2007).

[3] D. G. Yakovlev and C. J. Pethick, Annu. Rev. Astron. Astrophys. 42, 169 (2004).

[4] J. Pochodzalla, Prog. Part. Nucl. Phys. 39, 443 (1997).

[5] P. J. Siemens, Nature (London) 305, 410 (1983).

[6] P. Danielewicz et al., Science 298, 1592 (2002).

[7] C. B. Das et al., Phys. Rep. 406, 1 (2005).

[8] J. Pochodzalla et al., Phys. Rev. Lett. 75, 1040 (1995).

[9] J. B. Natowitz et al., Phys. Rev. C 65, 034618 (2002).

[10] L. G. Sobotka, R. J. Charity, J. Toke, and W. U. Schröder, Phys. Rev. Lett. 93, 132702 (2004).

[11] S. D. Gupta, A. Z. Mekjian, and M. B. Tsang, Advances of Nuclear Physics 26, Eds. J. W. Negele and E. Vogt (Springer, 2001).

[12] S. Levit and P. Bonche, Nucl. Phys. A437, 426 (1985).

[13] M. Baldo, L. S. Ferreira, and O. E. Nicotra, Phys. Rev. C 69, 034321 (2004).

[14] H. Jaqaman, A. Z. Mekjian, and L. Zamick, Phys. Rev. C 27, 2782 (1983).

[15] J. Kapusta, Phys. Rev. C 29, 1735 (1984).

[16] A. L. Goodman, J. I. Kapusta, and A. Z. Mekjian, Phys. Rev. C 30, 851 (1984).

[17] H. Müller and B. D. Serot, Phys. Rev. C 52, 2072 (1995).

[18] Guo Hua, Liu Bo, and M. DiToro, Phys. Rev. C 62, 035203 (2000).

[19] T. Sil, S. K. Samaddar, J. N. De, and S. Shlomo, Phys. Rev. C 69, 014602 (2004).

[20] P. Chomaz, M. Colonna, and J. Randrup, Phys. Rep. 389, 263 (2004).

[21] A. Lejeune, P. Grangé, M. Martzolff, and J. Cugnon, Nucl. Phys. A453, 189 (1986).

[22] M. Baldo and L. S. Ferreira, Phys. Rev. C 59, 682 (1999).

[23] A. Rios, A. Polls, A. Ramos, and I. Vidaña, Phys. Rev. C 72, 024316 (2005).

[24] B. Friedman and V. R. Pandharipande, Nucl. Phys. A361, 502 (1981).

[25] M. Baldo, I. Bombaci, L. S. Ferreira, G. Giansiracusa, and U. Lombardo, Phys. Lett. B215, 19 (1988).

[26] V. Soma and P. Bozek, Phys. Rev. C 74, 045809 (2006).

[27] A. L. Fetter and J. D. Walecka, Quantum Theory of ManyParticle Systems (Dover, New York, 2003).

[28] H. R. Moshfegh and M. Modarres, Nucl. Phys. A749, 130 (2005).

[29] H. Kanzawa, K. Oyamatsu, K. Sumiyoshi, and M. Takano, Nucl. Phys. A791, 232 (2007).

[30] A. Mukherjee and V. R. Pandharipande, Phys. Rev. C 75, 035802 (2007).

[31] W. Kohn and J. M. Luttinger, Phys. Rev. 118, 41 (1960).
[32] C. Bloch and C. de Dominicis, Nucl. Phys. 7, 459 (1958).

[33] C. Bloch and C. de Dominicis, Nucl. Phys. 10, 181 (1959).

[34] C. Bloch and C. de Dominicis, Nucl. Phys. 10, 509 (1959).

[35] B. terHaar and R. Malfliet, Phys. Rev. Lett. 56, 1237 (1986).

[36] H. Huber, F. Weber, and M. K. Weigel, Phys. Rev. C 57, 3484 (1998).

[37] L. P. Kadanoff and G. Baym, Quantum Statistical Mechanics (Benjamin, New York, 1962).

[38] R. D. Mattuck, A Guide to Feynman Diagrams in the Many-Body Problem (Dover, New York, 1992).

[39] W. H. Dickhoff and C. Barbieri, Prog. Part. Nucl. Phys. 52, 377 (2004).

[40] A. Schnell, T. Alm, and G. Röpke, Phys. Lett. B387, 443 (1996).

[41] T. Frick and H. Müther, Phys. Rev. C 68, 034310 (2003).

[42] T. Frick, H. Müther, A. Rios, A. Polls, and A. Ramos, Phys. Rev. C 71, 014313 (2005).

[43] P. Bozek and P. Czerski, Phys. Rev. C 66, 027301 (2002).

[44] B. Vonderfecht, W. Dickhoff, A. Polls, and A. Ramos, Nucl. Phys. A555, 1 (1993).

[45] P. Bozek, Nucl. Phys. A657, 187 (1999).

[46] H. Müther and W. H. Dickhoff, Phys. Rev. C 72, 054313 (2005).

[47] A. Rios, A. Polls, A. Ramos, and H. Müther, Phys. Rev. C 74, 054317 (2006).

[48] A. Rios, Ph.D. thesis, University of Barcelona, 2007.

[49] T. Frick, Ph.D. thesis, University of Tübingen, 2004.

[50] G. Baym, Phys. Rev. 127, 1391 (1962).

[51] T. Frick, H. Müther, and A. Polls, Phys. Rev. C 69, 054305 (2004).

[52] A. Rios, A. Polls, and H. Müther, Phys. Rev. C 73, 024305 (2006).

[53] W. Zuo, Z. H. Li, A. Li, and G. C. Lu, Phys. Rev. C 69, 064001 (2004).

[54] R. Machleidt, F. Sammarruca, and Y. Song, Phys. Rev. C 53, R1483 (1996).

[55] R. B. Wiringa, V. G. J. Stoks, and R. Schiavilla, Phys. Rev. C 51, 38 (1995).

[56] C. J. Pethick and G. M. Carneiro, Phys. Rev. A 7, 304 (1973).

[57] G. M. Carneiro and C. J. Pethick, Phys. Rev. B 11, 1106 (1975).

[58] Y. Dewulf, W. H. Dickhoff, D. Van Neck, E. R. Stoddard, and M. Waroquier, Phys. Rev. Lett. 90, 152501 (2003).

[59] P. Bozek and P. Czerski, Eur. Phys. J. A 11, 271 (2001).

[60] H. Müther and A. Polls, Prog. Part. Nucl. Phys. 45, 243 (2000).

[61] C. Das, R. K. Tripathi, and R. Sahu, Phys. Rev. C 45, 2217 (1992).

[62] E. Suraud, La Matière Nucléaire: des étoiles aux noyaux (Hermann, Paris, 1998).

[63] T. Frick, K. Gad, H. Müther, and P. Czerski, Phys. Rev. C 65, 034321 (2002). 